\title{
Automated Quantitative Cytology Imaging Analysis System in Cervical Cancer Screening in Shanxi Province, China
}

\author{
Yan Dong ${ }^{1}$, Jigeng Bai ${ }^{1}$, Yuping Zhang ${ }^{1}$, Guangjie Shang ${ }^{1}$, Yan Zhao ${ }^{1}$, Sha $\mathrm{Li}^{1}$, Ning Yan $^{1}$, Sumei Hao ${ }^{1}$ \& \\ Wenjuan Zhang ${ }^{1}$
}

\begin{abstract}
${ }^{1}$ Shanxi Maternal and Child Health Care Hospital, No 13 Xinmin North Street, Xinhualing District, Shanxi Province, China

Correspondence: Bojana Turic, 2366 Nanaimo street, Vancouver, British Columbia, V5N 5E3, Canada. Tel: 1-604-961-2619. E-mail: bojana.turic@gmail.com
\end{abstract}

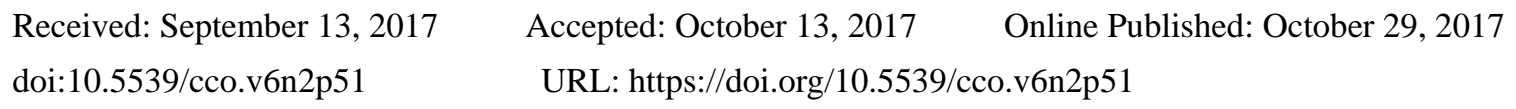

\begin{abstract}
Purpose: In China the number of pathologists is far from being enough to meet the demands of ongoing population based cervical cancer screening programs. This article aims to present our experience with automated quantitative cytology imaging platform, a reading system with an artificial intelligence that we currently use routinely for cervical cancer screening in Shanxi province.

Methods: From 2012-2016 a total of 40178 women were screened. Women were divided into three groups and each group had two subgroups. Smear and liquid based technique were compared using manual and automated platform.

Results: Detection rates of CIN2 + and positive rates of CIN2 were higher in all three groups when automated quantitative cytology platform was used compared with groups where reading was done by the pathologist using conventional microscope. Operator's costs associated with automated quantitative cytology platform vs. conventional reading using light microscope were compared too. The overall costs of operations based on automated platform were proven to be lower.

Conclusion: The use of automated platform and artificial intelligence as a means to overcome the lack of cytotechnologists and pathologists and to implement proper quality control in the large scale population based cervical cancer screening seems very promising.
\end{abstract}

Keywords: automated platform, quantitative cytology, cervical cancer screening, cytometry

\section{Introduction}

According to the WHO / IARC (World Health Organization / International Agency for Cancer Research) data from 2012 showed that cervical cancer is the fourth most frequent malignant tumor in women. The same report shows that the global annual incidence of cervical cancer was 528000 with estimated 266000 deaths in the same year. Out of that number about $85 \%$ of cervical cancer occurred in the developing countries, accounting for $12.0 \%$ of all female malignancies, while cervical cancer in developed countries accounted for only $3.0 \%$. The average age-standardized mortality rate for cervical cancer in developing countries was 8.3 per 100000 , while in developed countries that number is lower, at 3.3 per 100000 .

In China, cervical cancer is known to be one of the major malignant tumors that affects women's health and life. Peking University Health Care Center (2016) published that after 2000, the incidence of cervical cancer in China is on the rise while the mortality rate stayed somewhat the same. In 2015, the number of newly diagnosed cervical cancer cases was 98900 and the number of deaths reached 30500 . The prevalence of cervical cancer increased from 8.2/100 000 in 1999 to $15.1 / 100000$ in 2010 (Chinese Health Statistical Yearbook, 2011), while in Shanxi Province only, the prevalence of cervical cancer in 2015 was 57.66 / 100 000, representing the highest rate in the whole country. Detecting cervical precancerous lesions and implementing early screening followed by early treatment intervention are proven essential steps in prevention and treatment of cervical cancer. One of the key challenges in cancer control is a huge disparity between women from rural areas vs. those that live in the cities, in their awareness about screening program, willingness to participate or accesibility of proper care (Wang 2015). Therefore, exploring a simple and efficient screening method for cervical cancer that can be applied to 
screen a large-scale population of women has become our top priority. At the same time we aimed to find an economically sound approach.

\section{Materials and Methods}

\subsection{General Information}

From March 2012 to December 201640178 women were screened for cervical cancer and were divided into three groups. All women were 35-64 years old.

\subsubsection{Group 1}

Between 2015-2016, a total of 30124 rural women were enrolled into a screening program which was a part of Shanxi Province Initiative (Table 1). The sampling was done using cervical brush for either smears or liquid based cytology. In 21503 cases smear slides were stained with proprietary Feulgen DNA + EA50 staining. Semi automated quantitative cytology platform (further in the text Cyto System, Landing, Wuhan, China) with manual quality control was used to analyze slides and generate reports. The report used Bethesda (TBS) system reporting. In the same period specimens from another 8621 women were collected using liquid-based methodology, slides were stained by conventional PAP staining method and reading was done by pathologists using traditional microscopic examination. The same diagnostic Bethesda system (TBS) reporting was applied. Our goal was to compare detection rates between smears stained with DNA+EA50 and scanned using Cyto System scanning vs. conventional liquid based cytology.

Table 1. Rural women screened from 2015-2016

\begin{tabular}{llll}
\hline No. Women & Sample Preparation. & Staining & Reading \\
\hline 21503 & Smear & DNA+EA50 & Cyto System \\
\hline 8621 & Liquid based & Conventional PAP staining & Manual Microscope \\
\hline Total & & & \\
\hline
\end{tabular}

\subsection{2}

From October to December 2014 cervical specimens from 3253 rural women were obtained using cervical brush and from each sample two slides were made with liquid-based method (Table 2). One slide was stained with a proprietary Feulgen DNA+ EA50 staining, and scanned by Cyto System, while second slide was stained with traditional PAP staining, and analyzed by pathologist using standard microscopy method. Bethesda classification was applied for cytology diagnostic reporting. We wanted to compare two methods using the same sample, even though we were aware that there could be a difference between the slides in number and type of cells present.

Table 2. Group 2 Countryside women screened from October to December 2014

\begin{tabular}{llll}
\hline No.Women & Sample Preparation & Staining & Reading \\
\hline 3253 & Liquid based & Slide 1 DNA+EA50 & Cyto System \\
\cline { 3 - 4 } & & Slide 2 PAP & Manual Microscope \\
\hline
\end{tabular}

\subsection{3}

From 2013 to 2016, a total of 6981samples were collected from urban women using cervical brush (Table 3). All samples were processed using liquid-based preparation methodology. 2136 samples collected from 2013-2014 were stained with traditional PAP staining and conventional microscopy reading method by pathologists was done using Bethesda (TBS) classification for reporting. Samples collected from 2015 to 2016 from 4665 urban women teachers were taken during routine physical examination. Slides from these samples were stained with proprietary Feulgen DNA+EA50 staining protocol. Cyto System was used for scanning and report's generation. Bethesda system was used for reporting cytology diagnosis. In this group our aim was to compare two different methods for staining and analysis using the same sample preparation technique.

Table 3. Group 3 Urban, educated women screened from 2013- 2014

\begin{tabular}{ccccc}
\hline & No.Women & Sample Preparation & Staining & Reading \\
\hline & 4665 & \multirow{2}{*}{ Liquid based } & DNA+EA50 & Cyto System \\
\cline { 1 - 3 } \cline { 5 - 5 } & & & PAP & Manual Microscope \\
\cline { 1 - 1 } Total & 680136 & & & \\
\hline
\end{tabular}


In all three groups, women with positive results were invited for colposcopy. Colposcopic abnormalities were recorded and cervical biopsy was performed for histopathological diagnosis.

\subsection{Method}

\subsubsection{Cervical Sampling}

The chohort consisted of non-menstruating women, at least 3 days without vaginal medication or vaginal washing and no active sexual life. Professionally trained gynecologist took specimens under direct visualization of vagina using the standard Rovers' one-time sampling brush. The brush was rotated clockwise for 3-5 times at cervix (about $8 \mathrm{~mm}$ ). The goal was to collect sample from a cervical transition zone.

\subsubsection{Slide Preparation}

\subsubsection{Liquid Based Sample Preparation}

Sampling brush was detached and placed in the labeled collection jar containing standard fixative and send to the laboratory for further analysis. Cell deposition on the slide was done using Cytospin (Landing, Wuhan, China) which has round deposition area of $15 \mathrm{~mm}$ in diameter and surface area of 76, $72 \mathrm{~mm} 2$. See Figure 1.

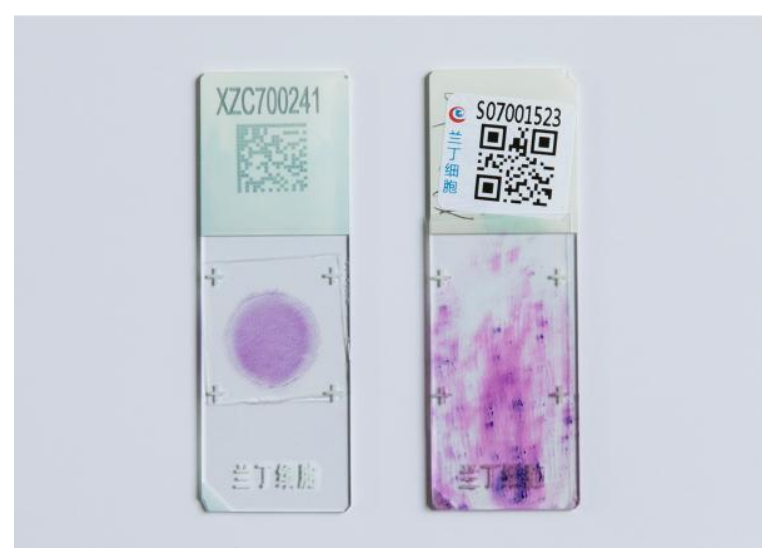

Figure 1. Liquid based and direct smear slide

\subsubsection{Direct Smear Method}

After sampling, specimen was placed on the slide by holding brush at 45 degrees angle in order to evenly apply sample on the slide, air drying was allowed for 15-20 seconds, after which slides were soaked into 95\% alcohol for 20 minutes for fixation, dried and were ready for staining.(Fig1)

\subsubsection{Laboratory Slide Staining}

Two staining protocols were used: proprietary staining Feulgen DNA+EA50 protocol in which Feulgen DNA stains nucleus first (blue), followed by counterstain EA50 which stains cytoplasm as a second step on the same slide. After fixation in $100 \%$ ethanol and Bounin solution, slides were hydrolized in 5N Hydrochloric acid for $60 \mathrm{~min}$ at $25{ }^{\circ} \mathrm{C}$, followed by staining with Thionin stain for $67 \mathrm{~min}$ at $25^{\circ} \mathrm{C}$. After rinsing, EA50 was applied for 2 seconds. Slides are then washed two times in $95 \%$ ethanol and 2 times in $100 \%$ ethanol before they were left to air dry and stored in the dark until image analysis. See Figure2. Second staining protocol was conventional routine PAP staining. 


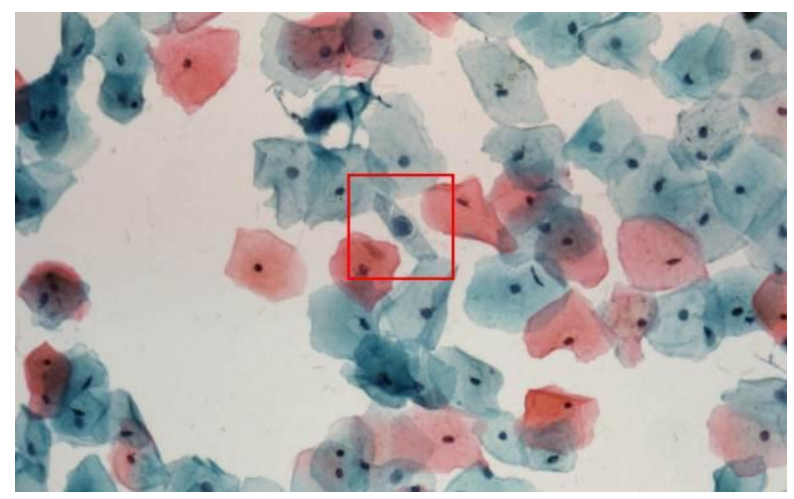

Figure 2. Visual field as seen under the light microscope

Nuclei are stained with Feulgen DNA (Thionin based) and cytoplasm is visualized applying EA 50.

\subsubsection{Slide Analysis and Reading}

Stained slides were scanned using Cyto System (Landing Med Tech, Wuhan), an automated quantitative cytology image analysis system. The system automaticaly selects the most suspicious objects, approximately 40 objects, based on more than 500 specific features. One of criteria is measurement of nuclear DNA content together with set of features for cell morphology recognition such as nuclear to cytoplasm ratio. This method was described and used previously (Guillaud et al., 2006) All scanned slides were first reviewed by trained technician who had 2-5 years of experience. Cervical cytology reports were classified according to Bethesda (TBS) diagnostic system and were reported as: ASCUS, ASC-H, LSIL, HSIL, cancer and other positive. All slides that were read as ASCUS and above were reviewed by pathologists and only $10 \%$ of randomly selected negative slides, as a part of quality control. Conventional cytology reading was done by pathologists who reviewed all the slides using standard light microscope.

Histopathology criteria: Biopsy diagnosis was graded as follows: normal, mild cervical dysplasia (cervicitis, CIN I), moderate and severe cervical dysplasia (CIN II and CIN III) and cervical invasive carcinoma.

\subsubsection{Follow Up}

According to the principle of "three steps" management: all women with ASCUS and above were examined by colposcopy, followed by biopsy if visual changes were observed. Colposcopy procedure required that the woman was abstained from sexual behavior, during the exam. Cervix was washed with saline, cervical secretions were carefully observed on the surface of the cervix, and then acetic acid staining (iodine test) was applied. Biopsy was taken from the suspicious area and sent to laboratory for histology reading.

\section{Results}

The first two groups were rural women while the third group consisted of urban more educated women where incidence of cervical cancer was known to be significantly lower. (Wang et al., 2015)

Table 4. Detection rates for Cyto System vs conventional microscopic reading method for positive cases, CIN 2 and above

\begin{tabular}{|c|c|c|c|c|c|c|}
\hline \multirow{3}{*}{ Groups } & \multirow{3}{*}{ Method } & \multirow{3}{*}{ No. of cases } & \multicolumn{2}{|c|}{ Cytology } & \multirow{2}{*}{\multicolumn{2}{|c|}{$\begin{array}{c}\text { Histopathology } \\
\text { CIN } 2+\text { CIN 3+Cancer }\end{array}$}} \\
\hline & & & \multirow{2}{*}{ No. of positive cases } & \multirow{2}{*}{ Positive rate } & & \\
\hline & & & & & Number of cases & Detection rate $/ 10^{4}$ \\
\hline \multirow{2}{*}{$1^{\text {st }}$ Group } & Cyto System & 21503 & 1065 & $4.95 \%$ & 236 & 109.75 \\
\hline & Manual & 8621 & 197 & $2.29 \%$ & 52 & 60.31 \\
\hline \multirow{2}{*}{$2^{\text {nd }}$ Group } & Cyto System & \multirow{2}{*}{3253} & $1^{\text {st }}$ slide 152 & $4.67 \%$ & 45 & 138.33 \\
\hline & Manual & & $2^{\text {nd }}$ slide 109 & $3.35 \%$ & 9 & 27.61 \\
\hline \multirow{2}{*}{ 3rd Group } & Cyto System & 4665 & 86 & $1.84 \%$ & 17 & 36.44 \\
\hline & Manual & 2136 & 25 & $1.17 \%$ & 3 & 14.05 \\
\hline
\end{tabular}

Out of total of 30124 women that participated, in the first group 21503 smear slides were analyzed using Cyto System, while in 8621 cases liquid based slides were sent for conventional manual slide reading done by pathologist using conventional light microscope. Although the sample preparation was not optimized for Cyto 
System we recorded 1065 positive cases (positive rate 4.95\%) (Table 4). Among those were 236 cases of CIN 2 and above including cases with cervical carcinoma. The detection rate was $109.75 / 10^{4}$. Out of 8621 cases whose samples were prepared with liquid based sample preparation and analyzed by pathologists using conventional microscope 197 cases were positive, positive rate of $2.29 \%$. Histology after biopsy found 52 cases of CIN 2 lesions and above (the detection rate was $60.31 / 10^{4}$ ).

A total of 3253 women participated in the second group. From each sample two slides were made using liquid based method at the same time. One slide was stained with Feulgen DNA+EA50 and scanned with Cyto System while the other slide was stained with conventional PAP stain and read manually by pathologist using light microscope. There were 152 positive cases (positive rate 4.67\%) in the Cyto System group, out of which 45 cases of CIN 2 above were found on histology. The detection rate was $138.33 / 10^{4}$. In a conventional manual reading group 109 positive cases were found (the positive rate 3.35\%) with only 9 cases of CIN 2 and above (the detection rate of $27.61 / 10^{4}$.)

A total of 6801 women participated in the third group. Out of 4665 cases whose slides were scanned using Cyto System 86 cases were positive, (positive rate 1.84\%), among those biopsy found 17 cases of CIN 2 and above lesions (the detection rate of $36,44 / 10^{4}$ ); manual reading in 2136 cases, found 25 cases positive, (positive rate $1.17 \%$ ). Among those pathological biopsy found 3 cases of CIN 2 and above (detection rate of $14.05 / 10^{4}$ ).

In all three groups the number of positive cases were significantly higher $(\mathrm{p}<0.05)$ if screening was done using Cyto System compared with a manual slide review by standard microscopy.

Positive rate comparison (X2 test) for Cyto System method of reporting please (Table 5). Note that the positive rate was significantly different in two groups.

Table 5. Comparison of positive cytology results among three groups of women

\begin{tabular}{cccccc}
\hline Group & Method & Positive & Negative & $\mathrm{X}^{2}$ & $\mathrm{P}$ \\
\hline \multirow{2}{*}{$1^{\text {St }}$ Group } & CS & 1065 & 20438 & 109.117 & $<0.05$ \\
& Manual & 197 & 8422 & & \\
$2^{\text {nd }}$ Group & CS & 152 & 3101 & 7.380 & $<0.05$ \\
& Manual & 109 & 3144 & & \\
$3^{\text {rd }}$ Group & CS & 86 & 4579 & 4.135 & $<0.05$ \\
\hline
\end{tabular}

It is not surprising that the overall number of positive cases was lower in a group of more educated of women when compared with the first two groups of rural women. It is observed by others, too, that urban women have easier access to cervical cancer testing, they are much more aware of the importance of regular screening and the incidence rate in this group is lower (Wang 2015).

It would be prudent to address the issue of sensitivity and specificity as well as positive predictive value of the method. Even though all women signed informed consent, the study lacks enough biopsies from negative women to properly calculate specificity, therefore these parameters are not presented. The authors are well aware of this insufficiency in their data.

3.1 Comparison of Costs between Automated Quantitative Cytology Platform Cyto System Analyses vs. Conventional Slide Reading

Costs were estimated from a provider point of view as a function of cost per positive case detected, based on detection rate we found in each group per 10000 women screened. During data collection only labor costs in each group were calculated. In China, conventional reading of slides by pathologists labor costs are approximately 30 yuan/case, while for automated scanner analysis (including labor costs and equipment ) the cost is 40 yuan/case. Colposcopic cost is, on average, about 60 yuan / case, cervical tissue biopsy approximately cost of 20 yuan / case, and cervical biopsy with histopathological diagnosis cost about 100 yuan / case (Table 6.) 
Table 6. Cost associated with manual and autiomated slide reading based on 10000 cases

\begin{tabular}{lllllll}
\hline & Manual & Cyto System & Manual & Cyto System & Manual & Cyto System \\
\hline Cost Manual & $30 * 10^{4}$ & 0 & $30 * 10^{4}$ & 0 & $30 * 10^{4}$ & 0 \\
CS cost & 0 & $40 * 10^{4}$ & 0 & $40 * 10^{4}$ & 0 & $40^{*} 10^{4}$ \\
Positive cases* & 229 & 495 & 335 & 467 & 117 & 184 \\
Colposcopy & $229 * 60$ & $495 * 60$ & $335 * 60$ & $467 * 60$ & $117 * 60$ & $184 * 60$ \\
Biopsy & $114.5 * 20$ & $247.5 * 20$ & $167.5 * 20$ & $233.5 * 20$ & $58.5 * 20$ & $92 * 20$ \\
Pathology & $114.5 * 100$ & $247.5 * 100$ & $167.5 * 100$ & $233.5 * 100$ & $58.5 * 100$ & $92 * 100$ \\
Total cost Per $10^{4}$ Screened & 327480 & 459400 & 340200 & 456040 & 314040 & 422080 \\
CIN2 and above** & 60 & 110 & 28 & 138 & 14 & 36 \\
Cost - effectiveness ratio C / E (RMB / case) & 5458 & 4176 & 12150 & 3304 & 22431 & 11724 \\
\hline
\end{tabular}

Note:

* The number of positive cases is obtained based on the positive rate calculated for each group

**The number of CIN2 is calculated based on detection rate for each group respectively

If we assume that the reading is done by conventional reading in 10000 cases that would amount to a total cost of $10000 * 30$ yuan $=300000$ yuan. (or using Cyto System 400000 respectively). In the first group according to calculated positive detection rate of $2.29 \%, 229$ cases (among 10,000) would need colposcopy. The cost of colposcopy at 60 Yuan will incur a total costs of $229 * 60=13740$ yuan. Further if we assume that $1 / 2$ of the cases sent to colposcopy will have biopsy, histopathological diagnosis will create additional costs of $114.5 *$ ( 20 +100 ) $=13740$ yuan. Finally the total cost would amount to $300000+13740+13740=327480$ yuan. (using Cyto System that would amount to 459400 yuan respectively) At this expense, with the detection rate of 60.31 we can assume that we should be able to detect 60 cases of CIN 2 and above and the would cost 5458 yuan per case (the cost-effectiveness ratio $(\mathrm{C} / \mathrm{E})=327480 / 60=5458$ yuan). At the detection rate for Cyto System of 109.75 the cost of 110 cases would be less expensive, 4176 yuan respectively.

The same principle is applied to all groups and the results are as expected, in each group using automated approach yield fewer costs overall.

This analysis has its limitations considering that the cost outcomes, training, overhead were not included. Despite these limitations our results show that it is possible to implement affordable technology and improve cancer detection at the same time.

\section{Discussion}

Cervical cytology has been around almost half a century and is still the main method for cervical cancer screening in several developed countries, such as the United States and Canada, regardless of the new (HPV) methodology that has been introduced in the last few years. Decades of screening data show that conventional cytology, can effectively reduce the incidence of cervical cancer and death rate (Barut et all, 2015) in a well organized cervical cancer program. In those developed countries more than $80 \%$ of women benefit from a regular cervical examination based on conventional cytology approach (Anderson, Boyes \& Benedet, 1988). Such practice has proved that cervical cytology is still, in health economic terms, very cost-effective method. However, in the developing countries due to lack of medical professionals (specially trained technicians and cytologists) and appropriate funding, many women, especially women in rural area or those with a low income are not receiving adequate medical care and are usually omitted from organized cervical cancer screening programs. Many of these women never had cervical examination in their life which is one of the main reasons why the incidence of cervical cancer in China is significantly higher when compared with incidence of cervical cancer in developed countries. According to the China Women's Development Program, published in 2009 Chinese government is conducting nationwide surveillance of cervical cancer, with the goal to screen $80 \%$ of women by 2020. ( Wang 2015) and these efforts are still ongoing.

In the developed countries where screening programs are well-established usually well-experienced cytotechnologist screen the slides first and is trained to issue negative results. All slides identified by cytotechnologist as potentially positive are reviewed by cytopathologists. In addition, pathologist reviews $10 \%$ to $20 \%$ of randomly selected negative cases as a part of quality control procedure. Because cervical cancer is a low incidence disease, most of the cases in any laboratory are negative, approximately $95 \%$ of all cases. Therefore, trained cytotechnologists in developing countries bear the vast majority of basic laboratory work, save labor costs and ensure quality at the same time. However, in China due to lack of specially trained cytology technicians and technologists, all slides are read by licensed pathologists, who are, in the most cases, not specialized in cytology. 
China is currently experiencing a serious shortage of pathologists, and the number of those actively working as cytologists is far from being able to meet the large population need for country's wide cervical cancer screening.

We used the same reading principle, cytotechnologist \& pathologist however we added Cyto System to help technician and pathologists in analyzing slides. This was possible due to the progress of science and technology, especially the emergence of computers, digital cameras and intelligent computing, automated and artificial intelligent diagnostic techniques that are currently available. Several systems that offer automation are already available in China, such as Thinprep diagnostic system by Hologic or Focal Point by BD. Both systems mark areas of interest that operator (techician or pathologist) needs to look at. While the image is improved by liquid based sample preparation the reading of the slides follows conventional reading principles. Education and experience of the reader still plays a major role in accurate reading. We used an automated quantitative cytology digital cell analyzer, Cyto System, that has two important features. It marks the area of interest and displays quantitative characteristic of each nucleai (cell). All captured objects are sorted in several categories and displayed on the computer monitor. Cytotechnician and or pathologist can further review each cell manually, in other words the system is used interactively although it is capable of generating report automatically. Displayed cells are selected for review based on quantitative measurements (over 500 features per cell) which is possible due to DNA stoichiometric staining, a true quantitative staining. Cytoplasmic EA50 staining on the other hand is secondary stain and its role is to add several features to overall automated analysis but mainy to support visualization of cytoplasm and that has a role to help reviewer .These quite unique features (Garner, 2014) distinguish Cyto System from other available automated reading systems. Combination of measurement and qualitative image (DNA content and cell morphology) assists operators, specially those less experienced to achive the same results as the most experienced one, minimizing human error and standardizing the report.

Currently available automated systems are designed only to display regions of interest that reviewers need to look at, usually 20 different fields, and to authors knowledge they are not capable of true measurement of nuclear DNA. The automated quantitative imaging platform in our study achieved both functions : measure of nuclear features (DNA content) and cytoplasmic visualization, both of which contribute to facilitate easier and more reliable reading.

Data collected from all our groups where slides were scanned using Cyto System showed that using automated quantitative imaging platform identified more cytology positive cases (and CIN 2 lesions) when compared to the traditional microscopic ( manual) diagnosis performed by pathologist.

In the future the gap between artificial intelligence and manual readings will become increasingly larger in favor of the former. It is expected that the automated intelligent diagnostic systems will add to self-learning and self-improvement among operators since all displayed nucleai and cells are accompanied with measurements as well. The use of intelligent computer reading system as a substitute for the cytology technician will likely be a future trend in the development of cell pathology and logical evolution in this field in China.

Another possible benefit from the use of artificial intelligence is easier and faster professional training among those pathologists that are not specifically trained as cytologists. With that we can expect substantial improvement in quality control across many laboratories.

According to published literature the range for accuracy in detecting early cervical cancer between pathologists is 53.4\% to 94\% (Nanda et al. 2000 and Oh, Shin, Gong, Sohn \& Khang 2008) almost 30\% difference when diagnosis is made by conventional cytology. It is understandable since morphological examination done by standard microscopic examination have many limitations: firstly specimen limitation such as sampling, sample preparation, staining variations, lack of continuous staff training and cervical cancer cytology specific training to mention only few. The other important factor is uneven distribution of specially trained cytologists in China, as many gravitate towards big centers leaving smaller hospitals without cytology expertise. That translates into uneven distribution of knowledge in cytology across many hospitals and regions .(Wright 2007).

In China major public health projects for women and children have been implemented since 2009 with the goal to complete cervical cancer screening for 70 million women by the end of 2020 . Until today 60 million rural women were screened. Screening work is mostly done in rural areas at the primary care hospitals which traditionally lack appropriate equipment and professional staff. Occasionally, due to various factors, it is difficult to assure a good quality of work, leading to a possible human error, poor repeatability and a higher rate of missed diagnosis . Data from 2013 to 2015 from China's national project showed that precancerous lesions' detection rate were around $150 / 10^{4}$ which confirms our thoughts that there is a room for improvements. One of the Cyto System advantages is its ability to compensate for the human resources, it is scalable and brings standardization into laboratory operations. Automated system will allow scanning of large number of slides and reading in a very 
consistent manner. While positive cases will continue to be analyzed by pathologists vast majority of negative slides can be scanned and reviewed by technician solving human bottleneck in cervical screening program in China. Automated quantitative cytology platform offers measurement information to pathologist, which can improve their individual performance too.

In North America and Europe, similar automated methods have been adopted as a routine laboratory testing method in cervical cancer screening programs quite successfully (Wright 2007 and Li, Zhang, \& Li 2010).

The operating cost for processing and analysing one sample using automated system is higher if we only take calculation for one case when it is compared with manual reading approach. However, using automated system we were able to detect more positive cases and due to the automated scanning process and its ability to scan slides overnight it seems more economic in a long run. Regarding the costs our data look promising and it seems possible to implement automated quantitative cytology platform at the reasonably low cost. It was published (Nghiem et all. 2015) previously that DNA ploidy can be economically beneficial and suggested that this approach could be easily implemented in everyday practice particularly in China. It is worth exploring this approach further for massive, population based screening projects.

\section{Conclusion}

We believe that combination of internet and automated artificial intelligence as a new approach to cervical cancer screening in China should be used particularly in a resource-deficient regions for cervical cancer screening as a first line method. With that approach we can achieve several beneficial effects for patients as well as for authorities that organize screening projects.

\section{Acknowledgment}

Authors would like to thank Dr. Linhong Wang from Chinese Center for Disease Control and Prevention for her guidance and support.

\section{References}

Anderson, G. H., Boyes, D. A., \& Benedet J. L. (1988). Organization and results of the cervical cytology-screening program in British Columbia, 1955-1985. British Medical Journal, 296(6627), 975-8. https://doi.org/10.1136/bmj.296.6627.975

Barut, M. U., Kale, A., Kuyumcuoglu, U., Bozkurt, M., Agacayak, E., ... Gul, T., (2015) Analysis of sensitivity, specificity, and positive and negative predictive values of smear and colposcopy in diagnosis of premalignant and malignant cervical lesions. Med Sci Monit, Dec 10, 21, 3860-3867. https://doi.org/10.12659/MSM.895227

Chinese Health Statistical Yearbook. (2011). Ministry of Health of the People's Republic of China. Beijing: Peking Union Medical College Press. (In Chinese)

Guillaud, M., Benedet, L. J., Cantor, B. S., Staerkel, G., Follen, M., \& MacAulay, C. (2006). DNA ploidy compared with human papilloma virus testing (Hybrid Capture II) and conventional cervical cytology as a primary screening test for cervical high-grade lesions and cancer in 1555 patients with biopsy confirmation.Cancer, 107(2), 309-318. https://doi.org/10.1002/cncr.21993

Garner, D. (2014). Clinical application of DNA ploidy to cervical cancer screening: A Review. World J Clin Oncol, 5(5), 931-965. https://doi.org/10.5306/wjco.v5.i5.931

Li, Z., Zhang, M., \& Li, H. (2010) Improved Detection of Cervical Cancer and High Grade Neoplastic Lesions by a Combination of Conventional Cytology and DNA Automated Image Cytometer. Journal of Cancer Therapy, 1, 47-51. https://doi.org/10.4236/jct.2010.12008

Nanda, K., McCrory, D. C., Myers, E. R., Bastian, L. A., Hasselblad, ... Matchar, D. B. (2000). Accuracy of the Papanicolaou test in screening for and follow-up of cervical cytologic abnormalities: a systematic review. Ann Intern Med., 132(10), 810-9. https://doi.org/10.7326/0003-4819-132-10-200005160-00009

Nghiem, V. T., Davies, K. R., Beck, J. R., Follen, M., MacAulay, C., ...Cantor, S. B. (2015) Economic evaluation of DNA ploidy analysis vs liquid-based cytology for cervical screening. British Journal of Cancer, 112, 1951-1957. https://doi.org/10.1038/bjc.2015.95

Oh, J. K., Shin, H. R., Gong, G., Sohn J. H., \& Khang, S. K. (2008). Diagnostic accuracy of conventional Pap test, liquid-based cytology and human papillomavirus DNA testing in cervical cancer screening in Korea: A meta-analysis. Korean Journal of Epidemiology, 30(2), 178-187. https://doi.org/10.4178/kje.2008.30.2.178

Davey, E., Barratt, A., Irwig, L., Chan, S. F., Macaskill, P., ... Saville, A. M. (2006). Effect of study design and 
quality on unsatisfactory rates, cytology classification, and accuracy in liquid-based versus conventional cervical cytology: a systematic review Lancet, 367(9505), 122-132.

https://doi.org/10.1016/S0140-6736(06)67961-0

Peking University Health Care Center. China Comprehensive Prevention and Control of Cervical Cancer. Beijing 2016 (in Chinese)

Wang, B., He, M., Chao, A., Engelgau, M. M., Saraiya, M., ... Wang, L. (2015). Cervical cancer screening among adult women in China, 2010. The Oncologist, 20, 627-634. https://doi.org/10.1634/theoncologist.2014-0303

WHO / IARC (World Health Organization / International Agency for Cancer Research, 2012

Wright, T. C. (2007). Cervical cancer screening in the 21st century: is it time to retire the PAP smear Clinical Obstetrics and Gynecology, 50(2), 313-323.

\section{Copyrights}

Copyright for this article is retained by the author(s), with first publication rights granted to the journal.

This is an open-access article distributed under the terms and conditions of the Creative Commons Attribution license (http://creativecommons.org/licenses/by/4.0/). 